GU J Sci, Part C, 6(3): 659-667 (2018)

Gazi Üniversitesi
Fen Bilimleri Dergisi
PART C: TASARIM VE TEKNOLOJI
dergipark.gov.tr/http-gujsc-gazi-edu-tr

\title{
Aynı Fiziksel ve Elektromanyetik Parametreler Altında EASMSM ve ORSMSM'un Vuruntu Torkuna göre Karşılaştırılması
}

\author{
Ali SAYGIN ${ }^{1, *}$, Ahmet AKSÖZ ${ }^{1}$ \\ ${ }^{I}$ Gazi Üniversitesi, Teknoloji Fakültesi, Elektrik-Elektronik Mühendisliği Bölümü, 06500, Yenimahalle/ANKARA
}

$\ddot{O} \mathbf{z}$

Makale Bilgisi

Başvuru: 01/03/2018

Düzeltme: 08/06/2018

Kabul: $13 / 06 / 2018$

Anahtar Kelimeler

EASMSM

ORSMSM

Vuruntu Torku

Keywords

AFPMSM

ORPMSM

Cogging Torque
Eksenel akılı sabit mıknatıslı senkron motor (EASMSM) ve outrunner sabit mıknatıslı senkron motor (ORSMSM) sıklıkla kullanılan döner tip motor çeşitleridir. Elektrik motorları için vuruntu torkunun istenmeyen bir çıktıdır. Yüksek devirlerde çalışırken vuruntu torku eylemsizlik momenti sayesinde önemli bir problem olmamasına rağmen, düşük devirlerde vuruntu torku üstesinden gelinmesi gereken bir ciddi sorundur. Özellikle bir uydu üzerindeki güneş panellerinin hareketi için vuruntu torku en aza indirilmelidir. Vuruntu torku sürekli mıknatıslı senkron motorların tasarımında çok önemli bir unsur olduğundan dolayı, bu çalışmada sadece vuruntu torkuna odaklanılmıştır. Bu nedenle, tasarlanan iki motor vuruntu torkuna göre karşılaştırılmıştır. Böylece en az vuruntu torku istenen uygulamalar için ne tip motorun tercih edilmesi gerektiği gösterilmiştir.

\section{Comparison of Axial Flux PMSM and Outrunner PMSM under Same Physical and Electrical Parameters according to Cogging Torque}

\begin{abstract}
Axial flux permanent magnet motor (AFPMM) and outrunner permanent magnet motor (ORPMM) are generic rotating machines that are commonly used. Cogging torque is an undesired output for electrical motors. Although the cogging torque is not an important problem at high operational speed thanks to moment of inertia, it is a serious trouble that must be overcome at low operational speed. Especially, it must be minimized for movement of solar panels on a satellite. Due to the fact that the cogging torque is so important factor for design of permanent magnet synchronous motor, in this study it is only focused on the cogging torque. For that reason, two designed motors are compared according to cogging torque. Thus, it has been shown what type of motor should be preferred for minimum cogging torque desired applications.
\end{abstract}

\section{GİRIŞ (INTRODUCTION)}

Havacılık uygulamalarında, elektrik motorları daha çok bozuntuların neden olduğu torkların sönümlenmesinde ve hızlı bir şekilde yönlendirilme işleminde kullanılmaktadır. Bu amaçla kullanılan motorlar, belirli bir atalete sahip olan tekerlek ünitesinin dönüş eksenini doğrultusunda tork üretmesi prensibine dayanmaktadır [1-3].

Yüksek hızlarda moment dalgalanmaları sistemin eylemsizliği sayesinde süzülebilir. Ancak, düşük hızlarda moment dalgalanmaları kabul edilemez boyutlarda hız değişimlerine, titreşime ve akustik gürültüye neden olur [4]. Vuruntu momenti sürekli mıknatıslardan kaynaklanan EMF harmonikleri ile statordaki oluklardan kaynaklanan manyetik iletkenlik harmoniklerinin etkileşiminden ortaya çıkar ve bir önlem alınmazsa motor miline ciddi boyutlarda yansıyabilir [1-2]-[5].

EASM motorlarda vuruntu momentini azaltmak için stator oluklarına ya da mıknatıslara eğim verilmesi (kaykı), mıknatıslara özel şekiller verilmesi ve kaydırılması, yardımcı olukların ya da dişlerin 
kullanılması, mıknatıs kutbunun optimizasyonu, kesirli sargıların kullanılması gibi birçok teknik uygulanmıştır [6-8].

Bu çalışmada, vuruntu torkunun EAPMSM ve ORPMSM nin aynı fiziksel ve elektromanyetik özellikleri altında karşılaştırılması çalışılmıştır. Bilgisayar simülasyonu sabit $3000 \mathrm{~d} / \mathrm{dk}$ nominal hız ve yüksüz motorlar ile yapılmıştır. $\mathrm{Bu}$ amaçla bölüm 2 de sabit mıknatıslı motorda vuruntu torkunun teorik teorik yapıs1 ve formulasyonu incelenmiştir. Sonlu elemanlar metodu tabanlı motor tasarımı bölüm 3de gerçekleştirilerek görsek ve grafiksel sonuçlar elde edilmiştir. Son olarak bölüm 4 de simulasyon sonuçları verilmiş, EAPMSM nin vuruntu torkuna göre daha iyi sonuç verdiği doğrulanmış ve yorumlanmıştır.

\section{SABIT MIKNASISLI MOTORDA VURUNTU TORKU(COGGING TORQUE IN PERMANENT MAGNET MOTOR)}

Tablo 1 de sabit mıknatıslı motorlarda vuruntu torkunu belirleyen değişkenler ve sabitler ifade edilmiştir.

Tablo 1. Vuruntu torkunu belirleyen değişkenler ve sabitler

\begin{tabular}{|c|c|}
\hline Değişken ve sabitler & Açıklaması \\
\hline$W_{m}$ & Mekanik enerji \\
\hline$F$ & Kuvvet \\
\hline$d_{x}$ & x yönündeki diferansiyel uzaklık \\
\hline$P_{m}$ & Mekanik güç \\
\hline$v$ & Hareket h1z1 \\
\hline$T$ & Tork \\
\hline$\varnothing_{g}$ & Hava aralığg akıs1 \\
\hline$\dot{d R}$ & Hava aralığının relüktansı değişimi \\
\hline$d \theta$ & Motor dönme açısı değişimi \\
\hline$T_{\operatorname{cog}}$ & Vuruntu torku \\
\hline$\theta_{m}$ & Rotor pozisyonu \\
\hline$\varphi_{k}$ & k inci harmoniğin genliği \\
\hline$N_{c}$ & $\mathrm{k}$ inci harmoniğin faz açıs1 \\
\hline$T_{k}$ & $\begin{array}{l}\text { Rotor kutup sayısı ile stator oluk sayısının en } \\
\text { kücük ortak katı }\end{array}$ \\
\hline$R_{m}$ & Miknatısın dış yarıçapı \\
\hline$R_{s}$ & Stator diş yarıçapı \\
\hline$R_{r}$ & Rotor yarıçap1 \\
\hline$\alpha p$ & Çift kutup sayısı \\
\hline$p$ & kutup adımı \\
\hline
\end{tabular}

Vuruntu momenti ister analitik ister sonlu elemanlar yöntemi ile belirlensin, Fourier serisi ile tanımlanabilir [9]. Tork dönme kuvvetinin bir ölçüsüdür ve motor tarafından üretilen tork tespit edilmesi gerek en önemli parametredir [10]. Mekanik bir sistemde enerjiyi iş olarak adlandırabiliriz. İși, ise mesafe ve kuvvetin bir ürünü olarak tanımlayabiliriz [11]. Diğer bir değişle iş, bir nesnenin yer değiştirmesidir ve yer değiştirmenin yönü boyunca kuvvetin bir bileşenidir. Böylece, mekaniksel enerjinin diferansiyel miktarı aşağıdaki gibi yazılabilir [12].

$$
d W_{m}=F d_{x}
$$

Burada $d W_{m}$ mekanik enerji, F kuvvet ve $\mathrm{dx}$ ise $\mathrm{x}$ yönündeki diferansiyel uzaklıktır. Güç, birim zamanda yapılan iş miktarı veya enerji değişiminin zamana oranıdır. Böylece mekanik enerji aşağıdaki gösterilebilir.

$$
P_{m}=\frac{d W_{m}}{d t}=F \frac{d_{x}}{d t}=\mathrm{Fv}
$$


Burada v hareket hızıdır. Hareket, dönme ile sınırlı olduğundan kuvvetten ziyade tork ile ilgilenilmelidir. Çevresel mesafe ile açısal pozisyon arasındaki ilişki $x=r \theta$ ile ifade edilir. Böylece mekaniksel güç,

$$
P_{m}=\frac{d W_{m}}{d t}=T \frac{d \theta}{d t}=T w
$$

bulunur.

Vuruntu torku, sabit mıknatıslı makinalarda rotor yüzeyine yerleştirilen mıknatıslar ve stator arasındaki manyetik etkileşimden kaynaklanmaktadır. Bu etki, sabit mıknatıslı makinalarda gürültü ve titreşime neden olduğu için istenmeyen bir durumdur. Vuruntu torkunun değeri aşağıda gösterilmiştir.

$$
T_{\operatorname{cog}}=\frac{1}{2} \emptyset_{g}^{2} \frac{d R}{d \theta}
$$

Burada $\emptyset_{g}$ hava aralığı akı değeri, $d R$ hava aralığının relüktansı ve $d \theta$ motor dönme açısını ifade etmektedir. Buradan da görüleceği gibi vuruntu torku mıknatıslarla (hava aralı̆̆ akı kaynağı) ve stator dişlerinin (değişken hava aralığı relüktansının kaynağı) etkileşimidir. Hava aralığının relüktansının periyodik olarak değişimi vuruntu torkunun da periyodik olarak değişimine neden olur. Bu periyodik değişimden dolayı vuruntu torkunun değeri fourier serisi ile hesaplanabilir.

$$
T_{\operatorname{cog}}\left(\theta_{m}\right)=\sum_{k=1}^{\infty} T_{k} \sin \left(k N_{c} \theta_{m}+\varphi_{k}\right)
$$

$\theta_{m}$ rotor pozisyonu, $T_{k} \mathrm{k}$ inci harmoniğin genliği, $\varphi_{k} \mathrm{k}$ inci harmoniğin faz açısı ve $N_{c}$ ise rotor kutup sayısı ile stator oluk sayısının en küçük ortak katını göstermektedir.

Vuruntu momenti hesabı için üç aşama vardır. Birinci aşamada, ideal oluksuz yapı için hava aralığındaki akı dağılımı formüle edilir. İkinci aşama da hava aralığı magnetik iletkenliği hesaba katılarak modifiye edilmiş hava aralığı akı yoğunluğu hesaplanır. Üçüncü ve son aşamada ise modifiye edilmiş hava aralığ 1 ak1 yoğunluğundan vuruntu momenti hesaplanır. Böylece ideal oluksuz makinenin stator yüzeyindeki ak1 yoğunluğu ifadesi aşağıdaki gibidir.

$$
\begin{gathered}
B_{r l}(\theta)=\sum_{n=1,3,5, \ldots}^{\infty} 2 \frac{\mu_{0} M_{n}}{\mu_{r}} \frac{n p}{(n p)^{2}-1}\left(\frac{R_{m}}{R_{s}}\right)^{n p+1}\left[\frac{(n p-1)+2\left(\frac{R_{m}}{R_{s}}\right)^{n p+1}-(n p+1)\left(\frac{R_{m}}{R_{s}}\right)^{2 n p}}{\frac{\mu_{r}+1}{\mu_{r}}\left[1-\left(\frac{R_{r}}{R_{s}}\right)^{2 n p}\right]-\frac{\mu_{r}-1}{\mu_{r}}\left[\left(\frac{R_{m}}{R_{s}}\right)^{2 n p}-\left(\frac{R_{r}}{R_{m}}\right)^{2 n p}\right] \cos (n p \theta)}\right. \\
M_{n}=2\left(\frac{B_{r}}{\mu_{0}}\right) \alpha_{p} \frac{\sin \left(\frac{n \pi \alpha_{p}}{2}\right)}{\frac{n \pi \alpha p}{2}}
\end{gathered}
$$

$R m$ mıknatısın dış yarıçapı, $R s$ stator dış yarıçapı, $R r$ rotor yarıçapı, $p$ çift kutup sayısı ve $\alpha p$ kutup adımidir.

\section{SONLU ELEMANLAR METODU TABANLI MOTOR TASARIMI (FINITE ELEMENT BASED MOTOR DESIGN)}

Literatürdeki kutup sayısı, oluk sayısı ve faz sayısı parametreleri ile yüksek devir ve yeterli bir tork üretebilecek bir tasarım yapılmıştır. Bu tasarıma ait parametreler Tablo 2 de verilmiştir. Burada tasarlanan motor reaksiyon tekeri olarak uydularda kullanılmaktadır.

Tablo 2. Kullanilan motor parametreleri

\begin{tabular}{|l|l|l|}
\hline \multicolumn{1}{|c|}{ Parametre } & \multicolumn{1}{c|}{ Değer } & \multicolumn{1}{c|}{ Birim } \\
\hline Kutup sayıs1 & 16 & \\
\hline Oluk sayıs1 & 24 & mm \\
\hline Hava aralığ1 & 1 & watt \\
\hline Sürtünme kayıpları & 10 &
\end{tabular}




\begin{tabular}{|l|l|l|}
\hline Sarım kayıpları & 20 & watt \\
\hline Referans hız & 4000 & rpm \\
\hline Anma çıkış gücü & 500 & watt \\
\hline Anma gerilimi & 48 & volt \\
\hline Anma hızı & 3000 & rpm \\
\hline
\end{tabular}

Tablo 1deki kutup sayısı oluk sayısı oranlarına göre 1mm hava aralıklı iki motor tasarımı Ansoft Maxwell RMxprt üzerinden yapılmıştır. Bu parametreler Rockwell Collins firmasının RSI 12 reaksiyon tekerine göre belirlenmiştir. Bu tekerin nominal hız değerinde motor torku $0.075 \mathrm{Nm}$ ve vuruntu torku tepe değeri en az 0.009 Nm olarak ölçülmüştür. Şekil 1 de RSI 12 görülebilir.

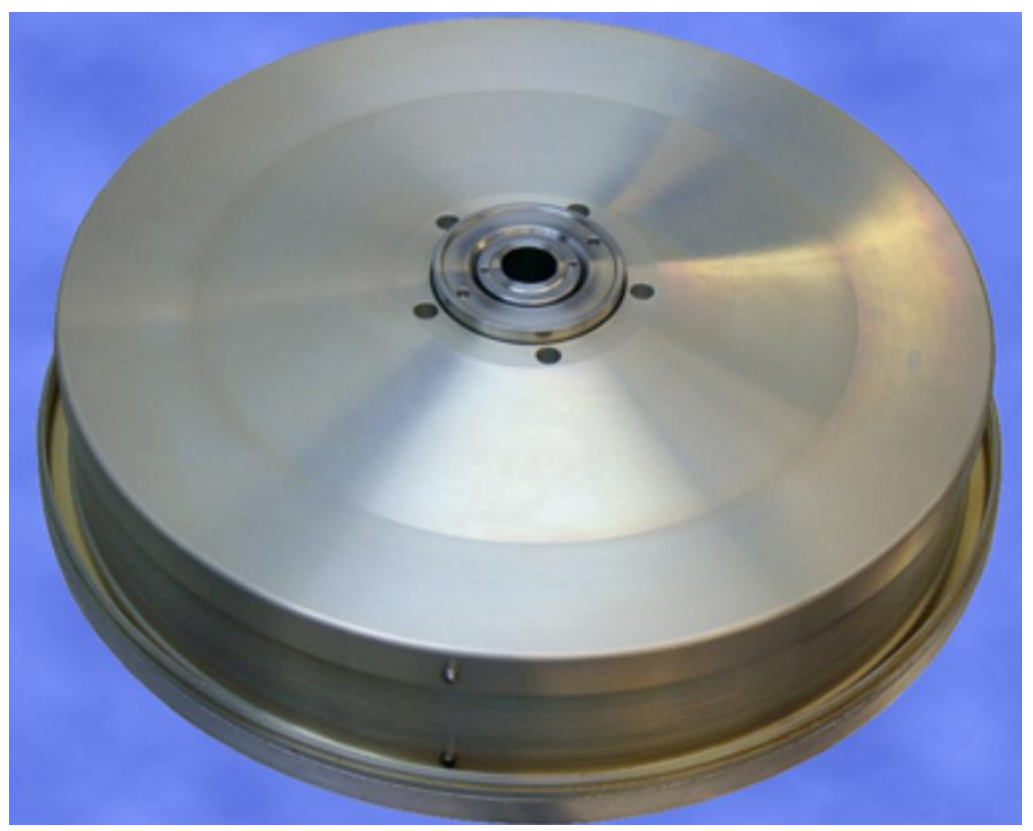

Şekil 1. Rockwell Collins RSI 12

Kayıplar, referans hız ve anma hızı, anma gücü ve anma gerilimi değerlerine göre Tablo 3 deki ve Tablo 3 deki stator ve rotor parametreleri de kullanılarak RMxprt deki tasarım Maxwell 3D arayüzüne geçirtilmiştir.

Tablo 3. Kullanilan stator parametreleri

\begin{tabular}{|l|l|l|}
\hline \multicolumn{1}{|c|}{ Parametre } & \multicolumn{1}{c|}{ Değer } & \multicolumn{1}{c|}{ Birim } \\
\hline D1ş çap & 180 & $\mathrm{~mm}$ \\
\hline İçap & 90 & $\mathrm{~mm}$ \\
\hline Uzunluk EAPMSM & 20 & $\mathrm{~mm}$ \\
\hline Uzunluk ORPMSM & 50 & $\mathrm{~mm}$ \\
\hline Malzeme & D23-50 & \\
\hline
\end{tabular}

Tablo 3 deki stator verilerine göre modellenen D23-50 malzemesinden stator gövdesi AFPMSM ve ORPMSM de motorun orta kısmındadır. Uzay koordinatlarına göre her iki motor içinde z ekseninde sabit bir gövde anlamına gelmektedir. Tablo 4 de motorların rotor parametreleri verilmiştir.

Tablo 4. Kullanilan rotor parametreleri

\begin{tabular}{|l|l|l|}
\hline \multicolumn{1}{|c|}{ Parametre } & \multicolumn{1}{c|}{ Değer } & \multicolumn{1}{c|}{ Birim } \\
\hline D1ş çap & 200 & $\mathrm{~mm}$ \\
\hline İç çap & 182 & $\mathrm{~mm}$ \\
\hline Uzunluk EAPMSM & 11 & $\mathrm{~mm}$ \\
\hline Uzunluk ORPMSM & 50 & $\mathrm{~mm}$ \\
\hline
\end{tabular}




\begin{tabular}{|l|l|l|}
\hline Malzeme & D23-50 & \\
\hline Miknatıs malzemesi & XG96/40 & \\
\hline Miknatıs kalınlığı & 4 & $\mathrm{~mm}$ \\
\hline
\end{tabular}

Tablo 4 deki verilere göre rotor tasarımı da yapılarak iki motor tasarımı da tamamlanmıştır. Aynı şekilde rotorlarda $\mathrm{z}$ ekseni üzerinde dönecek şekilde modellenmiştir. Hareket ekseni ortak olmasına rağmen, AFPMSM için daha kararlı çalışma ve dengeleme problemleri göz önüne alınarak çift rotorlu tasarım tercih edilmiştir [8]. Bölüm 4 de tasarlanan motorların simülasyon sonuçlarına yer verilmiştir.

\section{SIMULASYON SONUÇLARI(SIMULATION RESULTS)}

Bölüm 3 de Tablo 2, Tablo 3 ve Tablo 4 deki verilere göre oluşturulan RMxprt modeli Maxwell 3D arayüzüne aktarılarak motorlar görselleştirilmiştir. Şekil 2 de AFPMSM görülmektedir.

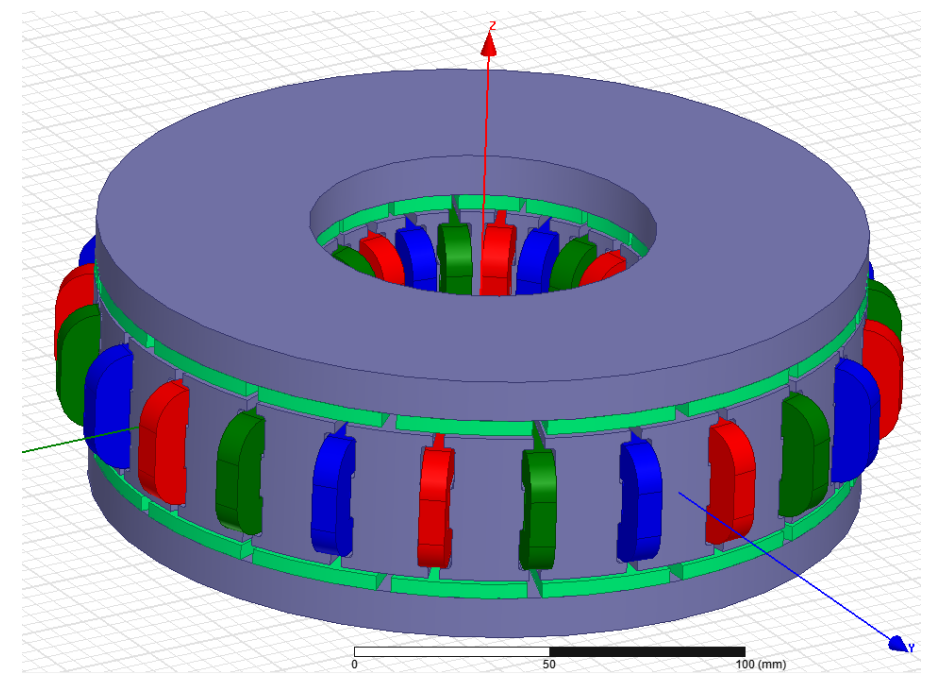

Şekil 2. Çift rotor eksenel akılı sabit mıknatıslı senkron motor 3D görüntüsü

Şekil 1 deki tasarıma ait veriler, rectengular plot edilerek sadece vuruntu torku analizi olmak üzere elde edilmiştir. Şekil 3 deki ORPMSM 3D görüntüsü gösterilmektedir. 


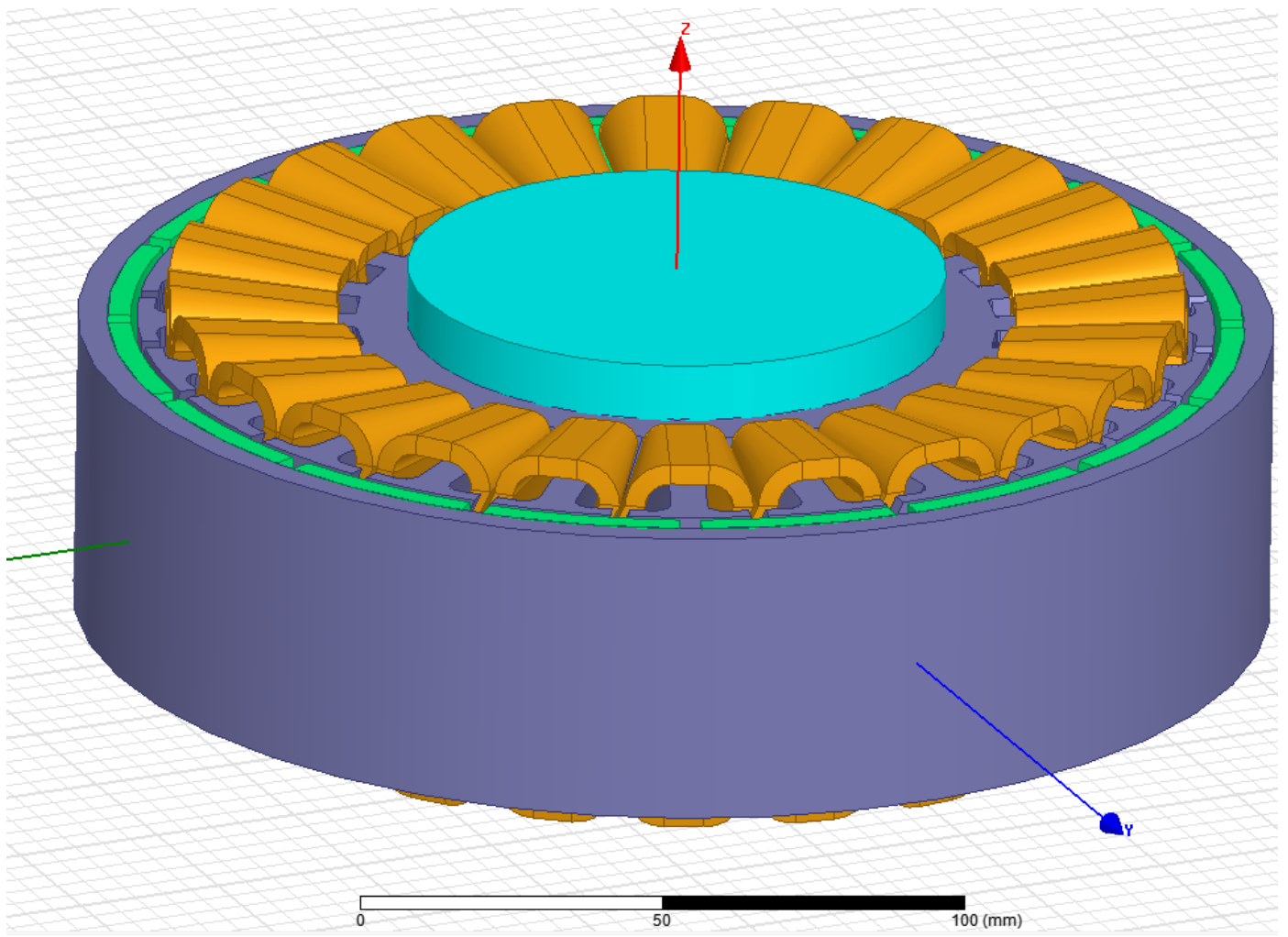

Şekil 3. Dış rotorlu sabit mıknatıslı senkron motor 3D görüntüsü

Şekil 3 de tasarlanan ORPMSM ile birlikte tasarım modelleri başarıyla gerçekleştirilmiştir. Şekil 4 de ise AFPMSM ait elde edilen vuruntu torku değerleri grafik üzerinden gösterilmiştir.

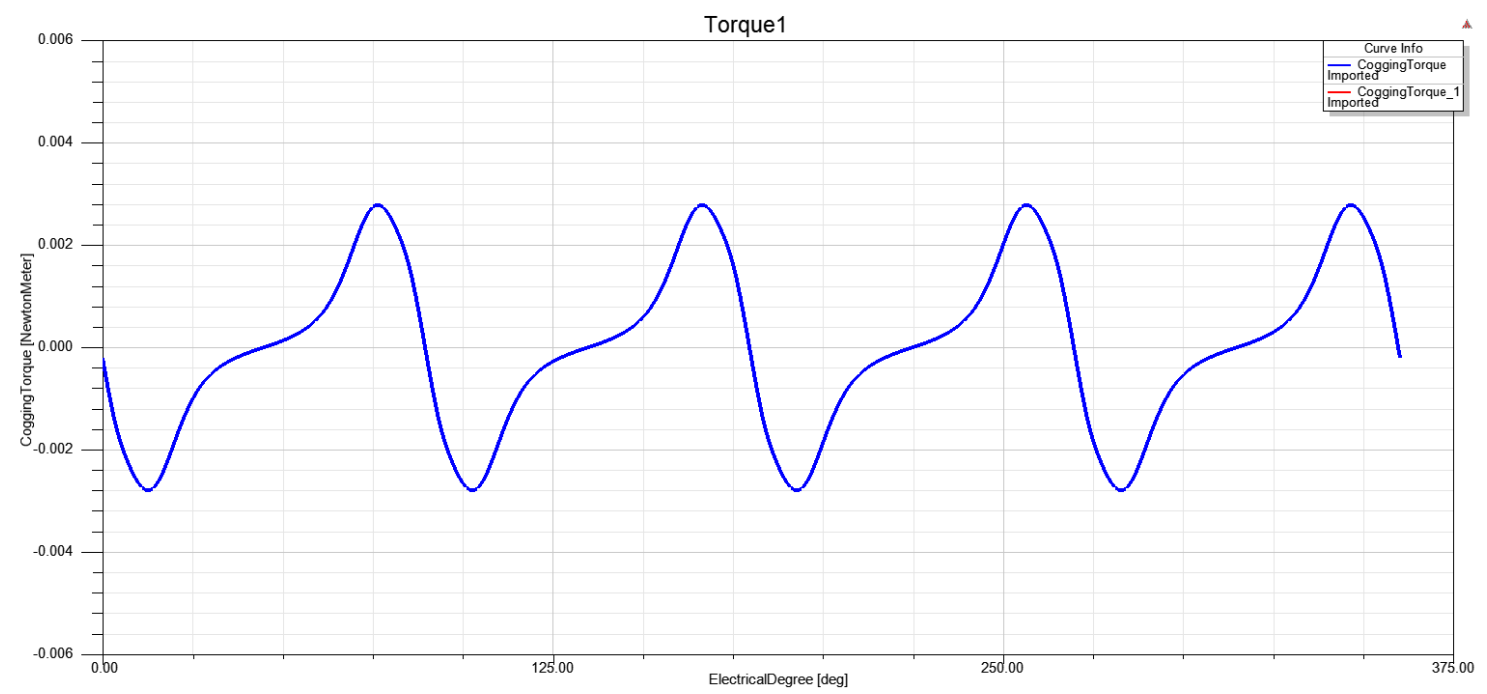

Şekil 3. AFPMSM ait vuruntu torku grafiği

Şekil 4 de görüldüğü gibi AFPMSM motora ait vuruntu torku değerleri $0.003 \mathrm{Nm}$ ile $-0.003 \mathrm{Nm}$ arasında değişmektedir. Şekil 5 de ORPMSM ait RMxprt ve Maxwell 3D de elde edilen vuruntu torku değerleri verilmektedir. 


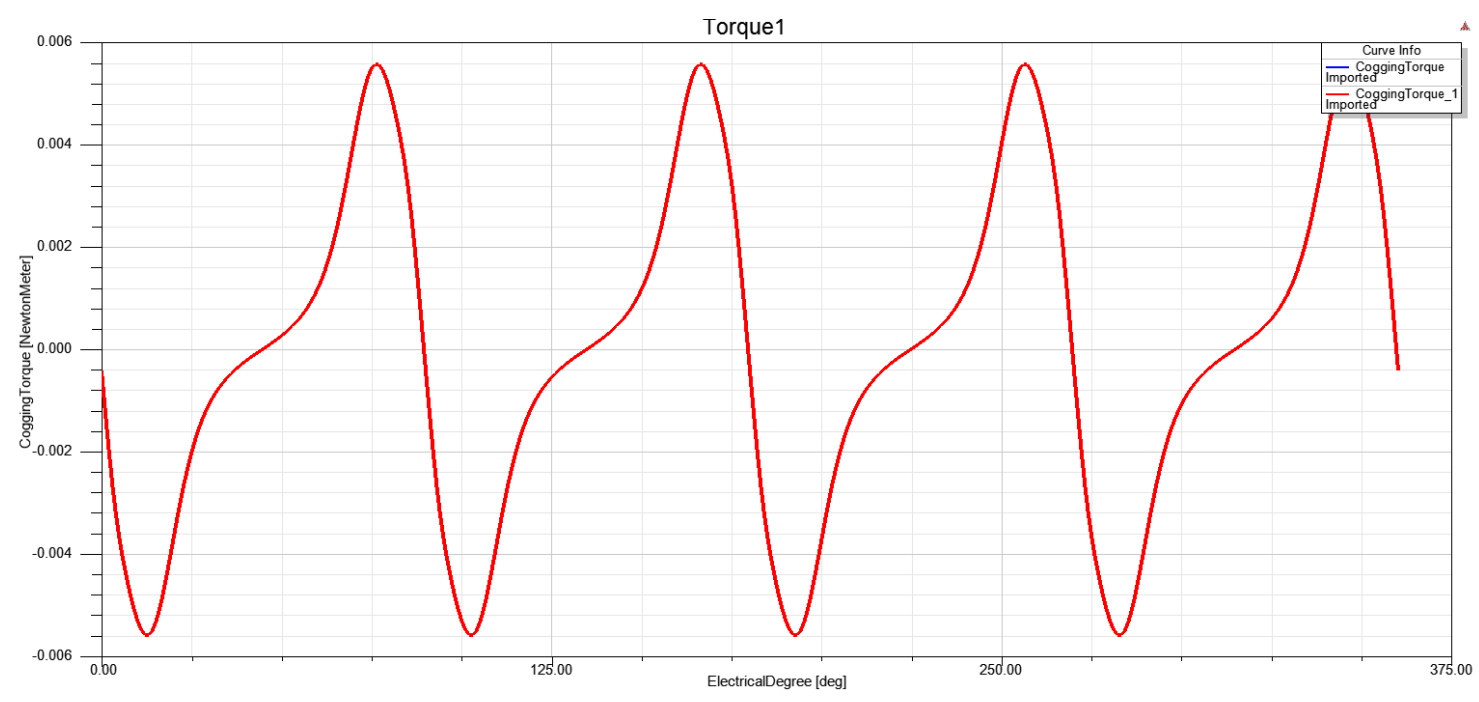

Şekil 4. ORPMSM ait vuruntu torku grafiği

Burada vuruntu torku $0.0056 \mathrm{Nm}$ ile $-0.0056 \mathrm{Nm}$ arasında salınım göstermektedir. Bu sonuçların sayısal karşılaştırmasını görselleştirmek için Ansoft Maxwell programında Şekil 4 deki değerler export edilerek bir csv dosyası elde edilmiştir. Daha sonra bu dosya Şekil 5 üzerinde import edilerek Şekil 6 deki vuruntu torku değerlerinin karşılaştırması ortak grafiği oluşturulmuştur.

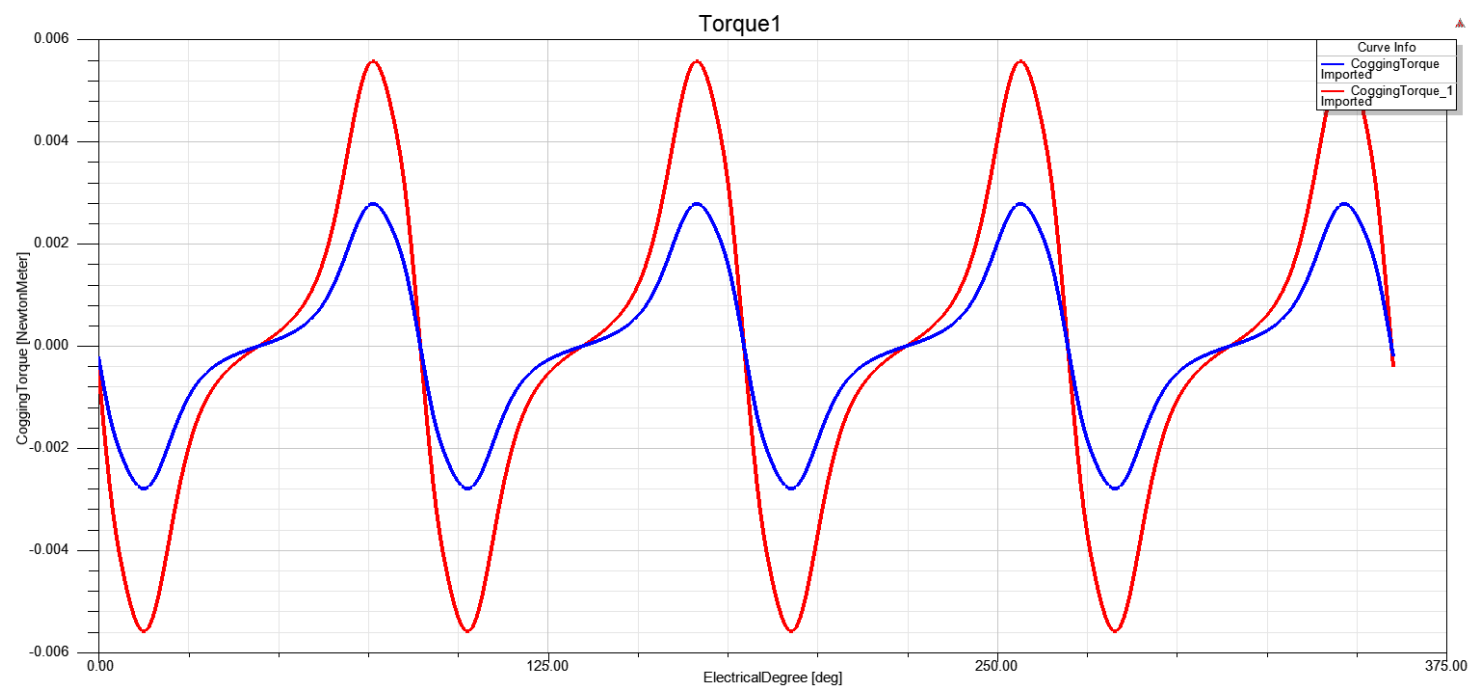

Şekil 5. ORPMSM ve AFPMSM ait vuruntu torklarının karşılaş̧ırılması grafiği

Şekil 6 göstermektedir ki aynı fiziksel ve elektromanyetik özelliklere sahip Şekil 2 deki AFPMSM ve Şekil 3 deki ORPMSM vuruntu torku değerleri farklılık göstermektedir. Bu farklılık AFPMSM ve ORPMSM nin çalışma prensiplerinden ortaya çıkmıştır. Bu farklılık göstermiştir ki vuruntu torkunun en az olması istenen uygulamalarda çift rotorlu AFPMSM kullanılması ORPMSM kullanılmasına göre daha avantajlıdır. Tablo 5 de sayısal olarak AFPMSM-ORPMSM vuruntu torku değerleri ifade edilmiştir.

Tablo 5. AFPMSM-ORPMSM vuruntu torku değerleri

\begin{tabular}{|l|l|l|}
\hline \multicolumn{1}{|c|}{ Motor } & $\begin{array}{c}\text { Tepeden tepeye vuruntu } \\
\text { torku (Nm) }\end{array}$ & \multicolumn{1}{c|}{$\begin{array}{c}\text { Vuruntu Torkunun } \\
\text { Nominal Torka oranı }\end{array}$} \\
\hline AFPMSM & 0.0060 & $\% 4$ \\
\hline ORPMSM & 0.0112 & $\% 7.5$ \\
\hline
\end{tabular}

\section{SONUÇ (CONCLUSION)}


Eksenel akılı sabit mıknatıslı senkron motor (EAPMSM) ve dış rotorlu sabit mıknatıslı senkron motor (ORPMSM) sıklıkla kullanılan döner tip motor çeşitleridir. Elektrik motorları için vuruntu torkunun istenmeyen bir çıktıdır. Yüksek devirlerde çalışırken vuruntu torku eylemsizlik momenti sayesinde önemli bir problem olmamasına rağmen, düşük devirlerde vuruntu torku üstesinden gelinmesi gereken bir ciddi sorundur. Özellikle bir uydu üzerindeki güneş panellerinin hareketi için vuruntu torku en aza indirilmelidir. Vuruntu torku sürekli mıknatıslı senkron motorların tasarımında çok önemli bir unsur olduğundan dolayı, bu çalışmada sadece vuruntu torkuna odaklanılmıştır. Bu amaçla, elektromanyetik analiz programında tasarlanan modeller vuruntu torkuna göre karşılaştırılmıştır. Böylece en az vuruntu torku istenen uygulamalar için ne tip motorun tercih edilmesi gerektiği gösterilmiştir. Bu çalışma sayesinde havacılık uygulamaları için vuruntu torkuna dikkat edilen yerlerde rotor pozisyonuna göre hangi motorun kullanılması gerektiği karşılaştırmalı olarak ifade edilmiştir. Böylece, EAPMSM nin vuruntu torkuna göre daha iyi sonuç verdiği doğrulanmış ve gösterilmiştir.

Daha sonraki çalışmada EAPMSM ve ORPMSM üretilerek gerçek motor ile tasarlanan motor arasında vuruntu torkları karşılaştırılacaktır.

\section{TEŞEKKÜR (ACKNOWLEDGMENTS)}

Bu çalışma TÜBİTAK 2211A tarafından desteklenmiştir.

\section{KAYNAKLAR (REFERENCES)}

[1] C. Kurtuluş, G. Inalhan, “Analysis of İTÜ NXG I Next Generation Technology Demonstrator from a Controls Perspective", Proc. Of 7th International ESA Conference on Guidance, Navigation \& Control Systems, Tralee, Ireland, 2008.

[2] N.K. Ure, Y.B. Kaya, G. Inalhan. "The development of a Software and Hardware-in-the-Loop Test System for ITU-PSAT II nano satellite ADCS." Aerospace Conference, 2011 IEEE. IEEE, 2011.

[3] A. Saygin, A. Aksöz, "Design optimization for minimizing cogging torque in Axial Flux Permanent Magnet machines," 2017 International Conference on Optimization of Electrical and Electronic Equipment (OPTIM) \& 2017 Intl Aegean Conference on Electrical Machines and Power Electronics (ACEMP), Brasov, 2017, pp. 324-329. doi: 10.1109/OPTIM.2017.7974991

[4] S. J. Arand, M. Ardebili, "Multi-objective design and prototyping of a low cogging torque axial-flux PM generator with segmented stator for small-scale direct-drive wind turbines," vol. 10, pp. 889-899, 2016.

[5] M. Thiele, S. Member, G. Heins, "Identifying cogging torque harmonics affected by misalignment in axial flux fractional pitch PMSM .," pp. 2969-2974, 2012.

[6] S. A. Afsari, H. Heydari, B. Dianati, "Cogging torque minimization in double sided axial flux magnetic gear," The 6th Power Electronics, Drive Systems \& Technologies Conference (PEDSTC2015), Tehran, 2015, pp. 71-76. doi: 10.1109/PEDSTC.2015.7093252.

[7] H. Tiegna, Y. Amara, G. Barakat, U. Havre, L. Havre, "Study of Cogging Torque in Axial Flux Permanent Magnet Machines Using an Analytical Model," vol. 50, no. 2, 2014.

[8] M. Gulec, M. Aydin, "Magnet asymmetry in reduction of cogging torque for integer slot axial flux permanent magnet motors," in IET Electric Power Applications, vol. 8, no. 5, pp. 189-198, May 2014, doi: 10.1049/iet-epa.2013.0256. 
[9] O. O. Ogidi, P. S. Barendse, M. A. Khan, "Influence of Rotor Topologies and Cogging Torque Minimization Techniques in the Detection of Static Eccentricities in Axial-Flux Permanent-Magnet Machine," in IEEE Transactions on Industry Applications, vol. 53, no. 1, pp. 161-170, Jan.-Feb. 2017, doi: 10.1109/TIA.2016.2616320.

[10] L. Xiao, J. Li, R. Qu, Y. Lu, R. Zhang, D. Li, "Cogging Torque Analysis and Minimization of Axial Flux PM Machines With Combined Rectangle-Shaped Magnet," in IEEE Transactions on Industry Applications, vol. 53, no. 2, pp. 1018-1027, March-April 2017, doi: 10.1109/TIA.2016.2631522.

[11] D. Lim, D. Woo, I. Kim, J. Ro, H. Jung, "Cogging Torque Minimization of a Dual-Type AxialFlux Permanent Magnet Motor Using a Novel Optimization Algorithm," vol. 49, no. 9, pp. 5106$5111,2013$.

[12] L. Xiao, J. Li, R. Qu, Y. Lu, R. Zhang, D. Li, "Cogging Torque Analysis and Minimization of Axial Flux PM Machines With Combined Rectangle-Shaped Magnet," in IEEE Transactions on Industry Applications, vol. 53, no. 2, pp. 1018-1027, March-April 2017, doi: 10.1109/TIA.2016.2631522 